\title{
EFFICACY OF PERITONEAL LAVAGE AND INTRAPERITONEAL INJECTION WITH CEFTRIAXONE COMBINATION WITH NEPHRECTOMY IN RENAL ABSCESS AND SEPTIC PERITONITIS FROM PROTEUS MIRABILIS IN A DOG
}

Piyabongkarn Damrongdej and Phalitakul Sukanya

Department of veterinary pharmacology, Faculty of veterinary medicine, Mahanakorn University, Bangkok, Thailand

\begin{abstract}
A dog showed urinary incontinence sign, anorexia, vomiting, and high fever. Radiographic revealed left kidney calculus, and enlargement kidney. A calculus with urinary bladder thickening was observed. Ultrasonography showed acoustic shadowing from the stone both left kidney and urinary bladder, and large anechoic area in a kidney. Abdominal surgery revealed pus from renal abscess rupture with fibrosis adhesion between renal and surrounding tissue. Cystotomy and nephrectomy were performed removing calculi. Peritoneal lavage with ceftriaxone was performed before suturing close of abdomen. Postoperative care with intraperitoneal injection of ceftriaxone was performed 5 day consecutively. A dog could be eaten and walk 2 days after operation. No septic peritonitis sign appeared after 30 days of operation. Proteus mirabilis was cultured from the kidney swab. The histological examination of kidney showed severe necrotic pyogranulomatous pyelonephritis. The excellent results revealed ceftriaxone by peritoneal lavage and intraperitoneal injection with nephrectomy surgery could be treated Proteus mirabilis septic peritonitis from renal abscess rupture.
\end{abstract}

Key words: ceftriaxone, renal abscess, septic peritonitis, Proteus mirabilis 


\section{INTRODUCTION}

Pyelonephritis generally refers to inflammation of the renal pelvis and adjacent renal parenchyma. Most cases of pyelonephritis are caused by an ascending bacterial infection (3). The other papers with perinephric abscess were found in some report papers $(6,1)$. Perinephric abscess is a rare and difficult to treat disease in veterinary medicine $(2,6)$. Renal abscess from Proteus mirabilis was very rarely uncommon cases in dogs. Renal abscess in a dog with diabetes mellitus was reported by Hss. R.S and Itan. I in 2006 that the first report of a renal abscess in a dog with diabetes (7). Proteus mirabilis, a causative agent that can infected normal renal change to renal abscess was rarely found in veterinary medicine. Bovillon. J. et al (2018) revealed retrospective histologically study dogs of 47 dogs with pyelonephritis that caused from E. coli and Staphylococcus spp in the most case (3). Colakoglu. E.C. et al (2017) studied efficacy of single-dose of ceftriaxone versus multiple-dose enrofloxacin in dogs with uncomplicated lower urinary tract infection and they concluded that ceftriaxone can be considered as alternative treatment of uncomplicated lower urinary tract infection (5). This is a report that showed successful treatment of renal abscess rupture with septic peritonitis from Proteus mirabilis by peritoneal lavage and intraperitoneal injection with ceftriaxone and nephrectomy surgery.

\section{Case description}

A twelve-year-old, shih-tzu dog was brought to clinic with high fever (105 F) and chronic urinary incontinence sign more than 2 years. A dog's clinical sign showed anorexia, vomiting, diarrhea, abdominal pain, and urinary incontinence sign.

\section{Radiography finding}

The radiography showed a large calculus in urinary bladder and a large calculus in a left kidney with left kidney enlargement (Fig 1,2)

\section{Ultrasonography finding}

In urinary bladder, an ultrasound scan showed a clear hyperechoic area with acoustic shadows in the urinary bladder. A size of urinary bladder calculus was measured about $2.5 \mathrm{~cm}$ (Fig3). A left kidney ultrasound scan revealed hyperechoic area with acoustic shadow in renal medulla and renal pelvis. A calculus was measured about $1.5 \mathrm{~cm}$. Anechoic area in left renal parenchyma was found from the scan. A left kidney was estimated about $4 \mathrm{~cm}$ (Fig 4).

\section{Hematological evaluation}

Before ceftriaxone and nephrectomy treatment, hematology revealed leukocytosis (23000 cells/mm3), normal in hepatic (14 units) and mild azotemia (creatinine $2.1 \mathrm{mg} \%$ ). Five days after treatment, hematology revealed no leukocytosis (14100 cells/mm3), and no azotemia (creatinine $1.5 \mathrm{mg} \%$ )

\section{Surgical procedure for cystotomy}

Ventral midline incision with cystotomy was performed for remove Calculus from the urinary bladder. The 
urinary bladder was sutured with polyglycolic acid 2/0(Fig 5,6 )

\section{Surgical procedure for left nephrectomy and removed adhesion tissue surrounding left kidney}

A dog (4.5 kg in body weight) was anesthetized with propofol $(6 \mathrm{mg} / \mathrm{kg})$ and maintained anesthesia with $2 \%$ isoflurane by anesthesia machine. Skin incision was performed at the region of the enlargement kidney, then abdominal muscles was incised and retracted until seen the kidney. Self-retaining retractor was used for separated the abdominal muscles. A left kidney appeared adhesion with surrounding tissue, including small intestines, omentum, peritoneal fat. Pus, fibrosis and hemorrhagic condition were found in the abdominal cavity. (Fig 7,8). Electrosurgical blade was used for dissection a left kidney free from surrounding adhesion tissues. A left kidney was removed by ligated renal artery, renal vein, and ureter by nylon $2 / 0$.

\section{Peritoneal lavage with ceftriaxone}

$1000 \mathrm{ml}$ NSS was mixed with ceftriaxone $1000 \mathrm{mg}$ for peritoneal lavage. After lavage finished, ceftriaxone $200 \mathrm{mg}$ was placed into abdominal cavity before closed the abdomen.

\section{Postoperative care}

A dog was treated with ceftriaxone $200 \mathrm{mg}$ twice a day by intraperitoneal route 5 days, consecutively, after operation. Supportive treatment with acetate fluid, $5 \%$ dextrose in water, tofenamic acid were used for control hydration status.

\section{Results and discussion}

\section{Clinical sign}

A dog could be eaten food after 2 days of operation and normal in activity. Five days after treatment, the hematologic parameters showed no azotemia, and normal blood leukocytes. The suture was removed 10 days after operation (Fig 9). One month after operation, septic peritonitis signs were not observed.

\section{Microbiology reports}

7 days after operation, Proteus mirabilis was cultured from a left kidney. Antibiotic sensitivity test showed Proteus mirabilis had susceptible with ceftriaxone, amoxycillin and clavulanic acid, cefotaxime, cephalexin, enrofloxacin, gentamicin, kanamycin, marbofloxacin, and meropenem. Proteus mirabilis in this case had resisted with amoxycillin.

\section{Pathology and histopathology report}

A left kidney was found $4 \mathrm{~cm}$ in size. Pus and $1.5 \mathrm{~cm}$ calculus were found in renal medulla and renal pelvis. The removed kidney was observed to had loss of renal parenchyma, severe hemorrhagic, and pus in renal parenchyma (Fig 10). Histopathology report by pathologist (Dr. Anudep Rungsipipat) revealed severe necrotic pyogranulomatous pyelonephritis and moderate membrano-proliferative glomerulonephritis (Fig 11).

The results revealed that septic peritonitis was treated with ceftriaxone by peritoneal lavage and intraperitoneal injection combined with nephrectomy. Ceftriaxone was directly used into septic region that 
had showed excellent efficacy for controlled peritonitis from Proteus mirabilis. Ceftriaxone was used for control septic peritonitis in human medicine but rarely used in veterinary medicine in septic peritonitis case. To the author's knowledges and my literature review, I cannot find intraperitoneal lavage by ceftriaxone combination with nephrectomy for successful treatment of renal abscess from Proteus mirabilis in dog. Renal abscess from Proteus mirabilis is very rare case in $\operatorname{dog}(1,2)$. Zatelli $A$. and $D^{\prime}$ Ippolito P. (2004) reported a failed treatment of bilateral perirenal abscesses in a cat (2). Pyelonephritis, perinephric abscess, and renal abscess have been reported in a little literature $(1,2,7)$. Bouillon et al (2018) used retrospective study of 47 histologically diagnosed cases (2005-2015). This report showed Escherichia coli was the most common pathogen isolated (37\%), only one case was pyelonephritis from Proteus mirabilis (3). Cancelinha. C et al
(2014) reported two uncommon case of renal abscess from Proteus mirabilis in childhood. Case 1 was treated by flucloxacillin with ceftriaxone. Case 2 was treated with amoxicillin-clavulanic acid and surgical drainage for 5 days with catheter (4). In this case, a dog was treated with out catheter's surgical drainage and ceftriaxone can destroyed Proteus mirabilis that correlated with antibiotic sensitivity test. The efficacy of ceftriaxone by peritoneal lavage and intraperitoneal injection route for controlling septic peritonitis from Proteus mirabilis renal abscess was not found from the other literatures.

\section{Conclusion}

Ceftriaxone administrated by peritoneal lavage and intraperitoneal injection had enough efficacy for controlled septic peritonitis due to Proteus mirabilis renal abscess in dog.

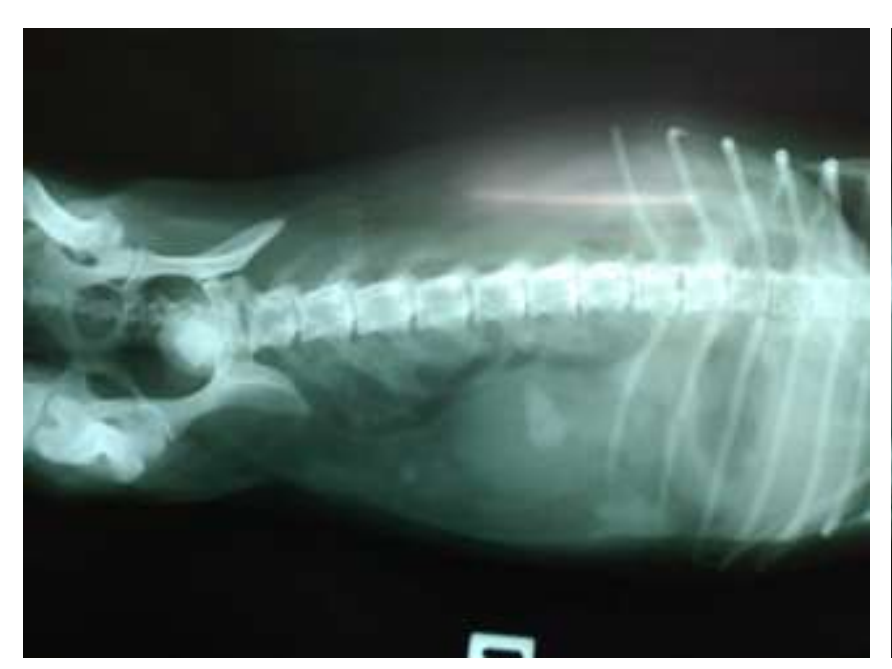

Fig:1

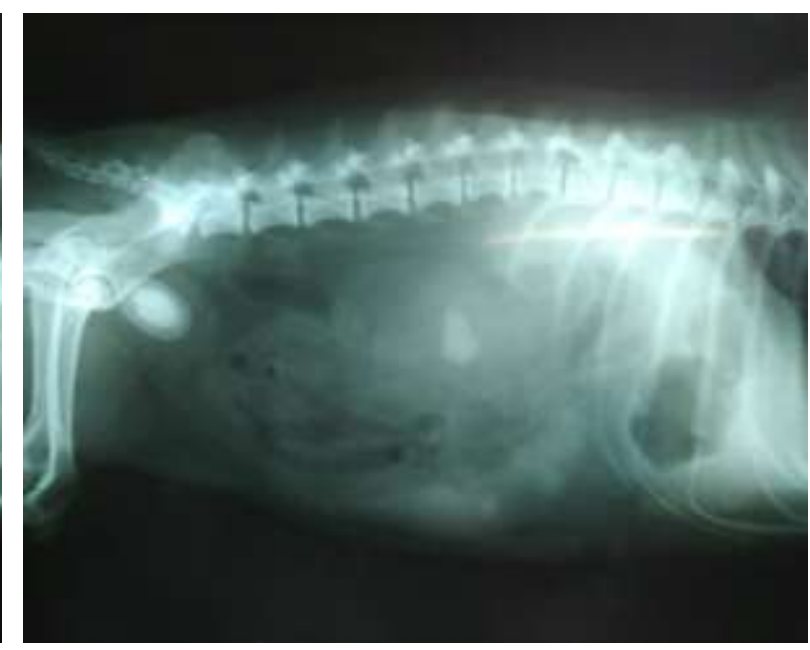

Fig : 2 


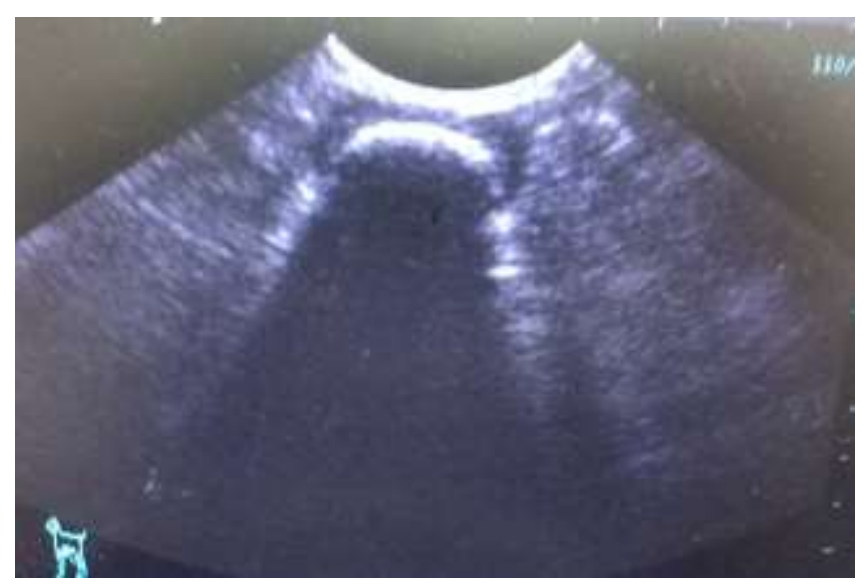

Fig: 3
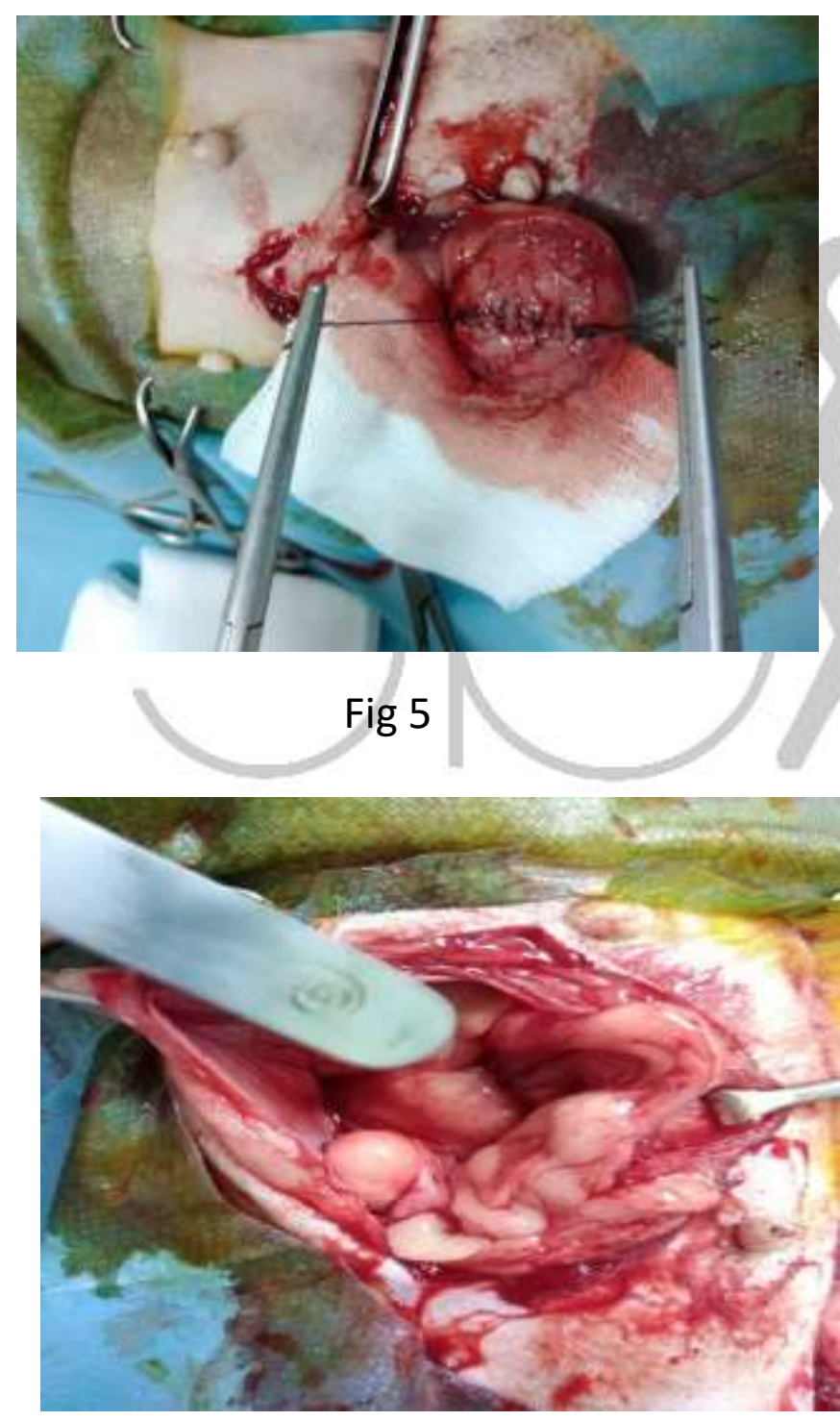

Fig : 7

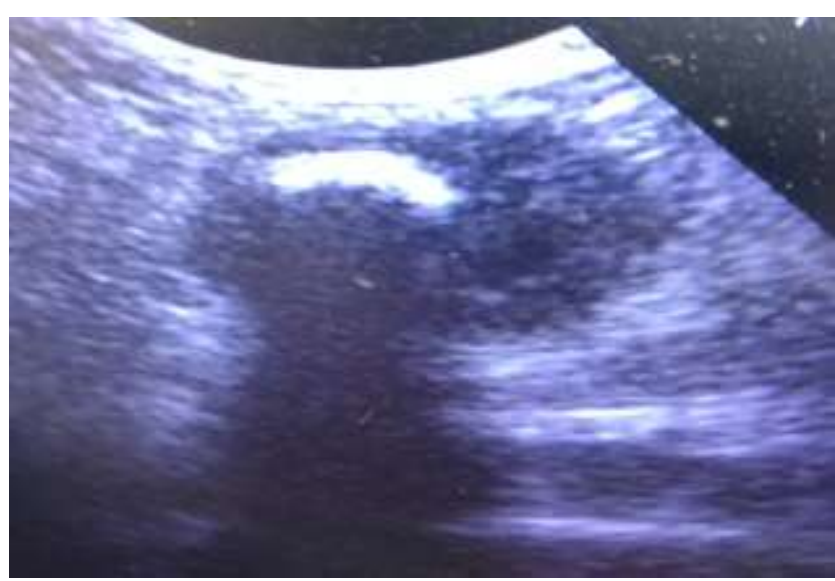

Fig: 4
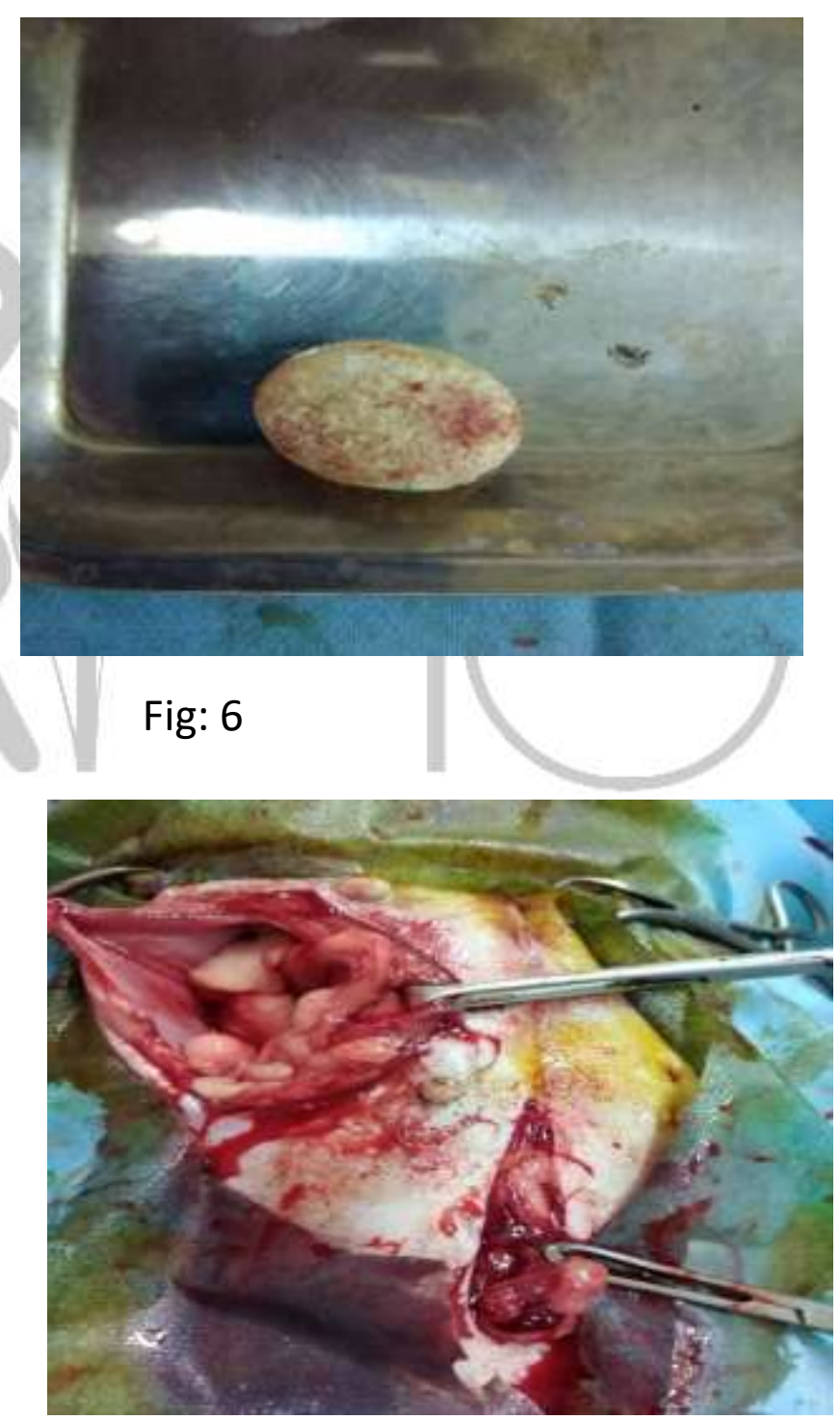

Fig : 8 


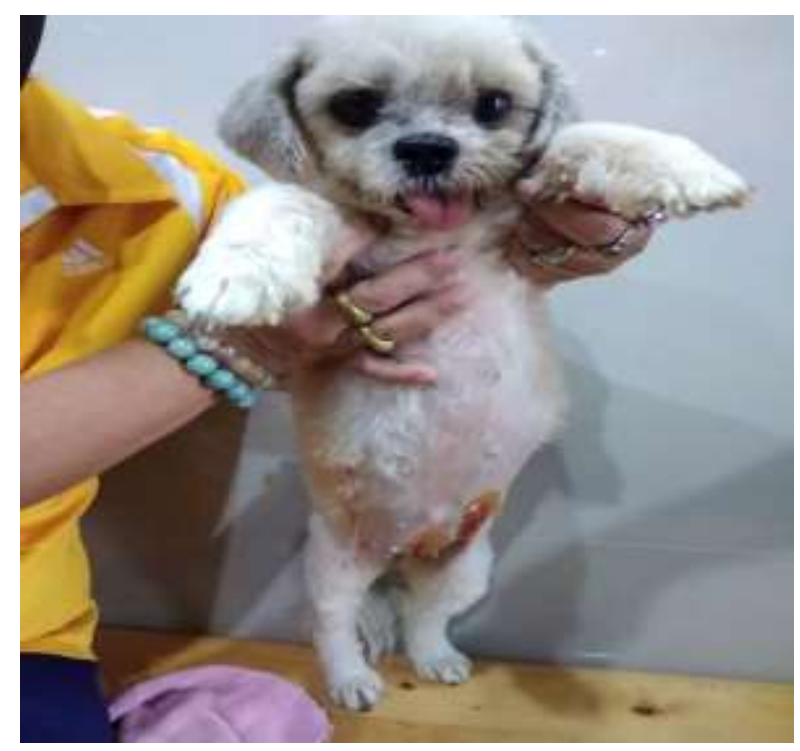

Fig : 9

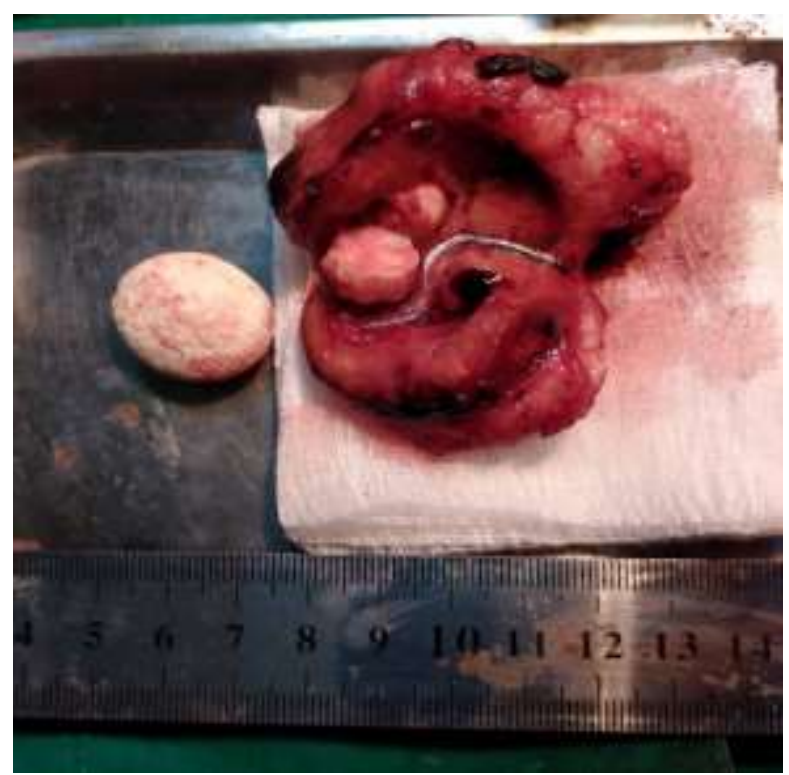

Fig : 10
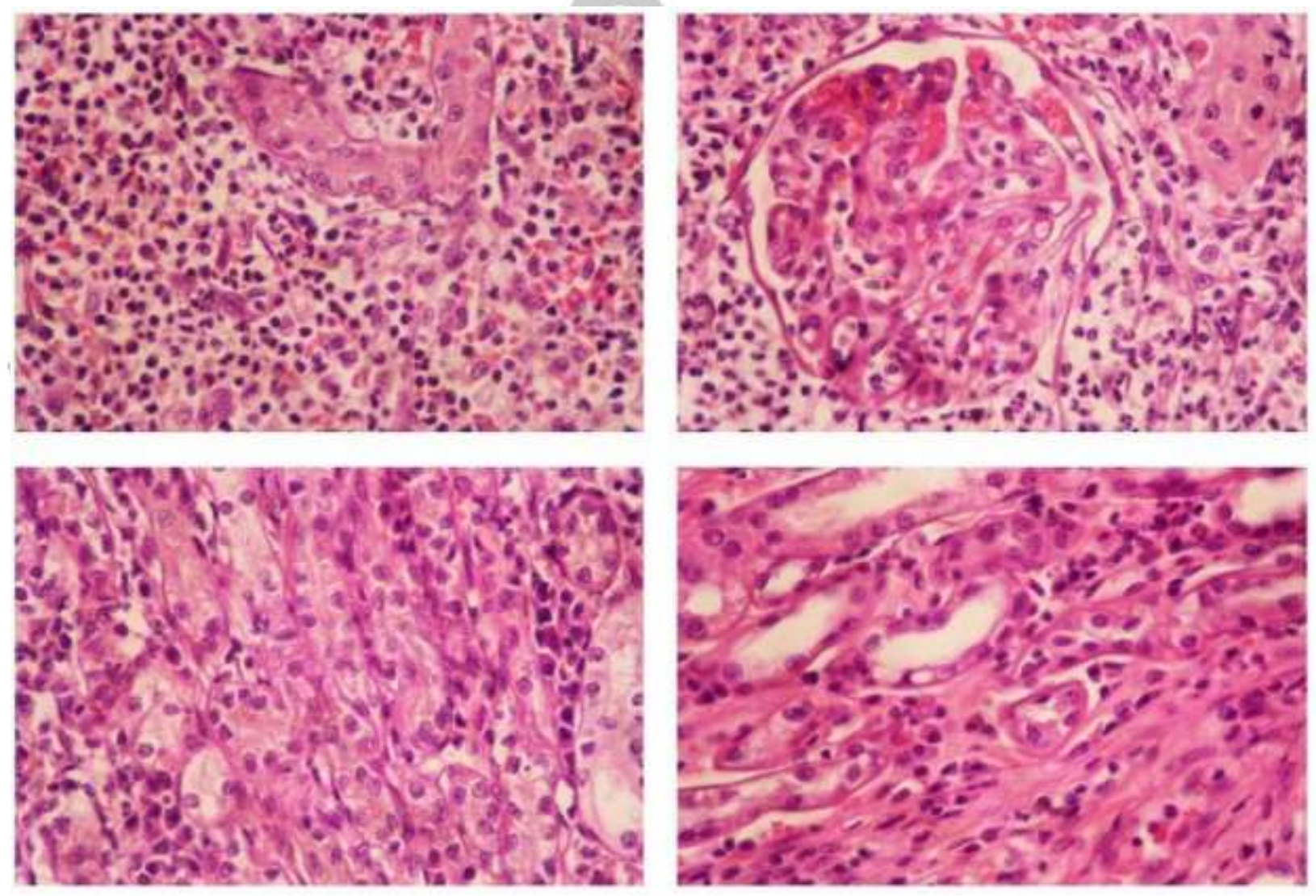

Fig: 11 
Figl Radiography (VD position) showed a large calculus in urinary bladder and left kidney.

A left kidney was a large size.

Fig2 Radiography (lateral position) showed a large calculus in urinary bladder and left

kidney.

Fig3 Ultrasonography showed a clear hyperechoic area with acoustic shadows in the urinary bladder.

Fig4 Ultrasonography showed a clear hyperechoic area with acoustic shadows in renal medulla and renal pelvis. An echoic area in a left renal parenchyma.

Fig5 Urinary bladder was sutured after removed calculus.

Fig6 A large calculus from urinary bladder

Fig7 Pus and blood exudate surrounded a large left kidney

Fig8 A inflammatory kidney adhered surrounding tissue and visceral organ.

Fig9 Sutures were removed after 10 days of operation. No septic peritonitis clinical sign.

Fig10 Pus exudate in the renal parenchyma with renal calculus and urinary calculus.

Figl 1 the figure revealed mild to moderate thickening of glomerular basement membrane and multifocal pyogranulomatous in interstitial area of cortex, medullar and renal pelvis. Each pyogranuloma consisted of necrosis, accumulation of segmented neutrophils, macrophages, lymphocytes and plasma cells. Multifocal areas of interstitial fibrosis were seen.

\section{References}

Agut, A., Laredo, F.G., Belda, E., Seva, J., Soler, M. (2004). Left perinephric abscess associated with nephrolithiasis and bladder calculi in bitch. The Veterinary record, 562565.

Zatelli, A., and D'Ippolito, P. (2004). Bilateral perirenal abscesses in a domestic neutered shorthair cat. J Vet Intern Med, 18, 902-903.

Bouillon, J., Snead, E., Caswell, J., Feng, C., Helie, P., Lemetayer, J. (2018). Pyelonephritis in dogs: retrospective study of 47 histologically diagnosed cases (20052015). J Vet Intern Med,32, 249-259.

Cancelinha, C, Santos, L., Ferreira, C., Gomes, C. (2014). Renal abscesses in childhood: report of two uncommon cases, 1-4.

Colakoglu, E. C., Haydardedeoglu, A. E., Alihosseini, H., Hayirli, A. (2017). Efficacy of single-dose ceftriaxone versus multipledose enrofloxacin in dogs with uncomplicated lower urinary tract infection: a randomized clinical trial. Veterinarni Medicina,62(3), 125-130.

Guedes, R.L., FDornbusch, P.T. et al. (2018). Renal capsulotomy associated with omentopexy for treatment of bilateral perinephric abscess in a bitch: case report. Ciencias Agrarias, Londrina,39(5), 23012306.

Hess, R. S., Ilan, I. (2006). Renal abscess in a dog with transient diabetes mellitus. Journal of small animal practice,44(1). 